Article publié dans : E. Moline \& C. Vetters (éds), Temps, aspect et modalité en français. Amsterdam : Rodopi, 2010, 1-23. (Cahiers Chronos 21)

\title{
La résultativité et la valeur de parfait en français et en polonais
}

\author{
Denis APOTHÉLOZ \\ Université de Nancy 2 et ATILF (UMR 7118) \\ Małgorzata NOWAKOWSKA \\ Université Pédagogique de Cracovie
}

\section{Introduction}

Cette étude comporte deux parties. La première aborde la question de la résultativité et de ses rapports avec le parfait. Elle a pour objectif principal de présenter le cadre conceptuel à partir duquel seront menées les analyses de la seconde partie. Nous y développons principalement deux idées. Tout d'abord, nous montrons que la bi-temporalité constitutive de la valeur de parfait est propice à l'expression de certaines inférences liant le contexte d'énonciation à une situation antérieure, ce qui nous conduit à distinguer trois types inférentiels de parfait. Ensuite, nous montrons que parmi les états résultants associés au parfait, il est utile de distinguer entre résultativité « sémantique » et résultativité " pragmatique ». Cette première partie aborde également la question du parfait d'expérience et présente une typologie des valeurs de parfait.

La seconde partie s'intéresse à l'expression de la résultativité en polonais. Après avoir présenté un rapide aperçu du système aspectuotemporel de cette langue, nous étudions les différents emplois qui y sont faits des formes verbales perfectives et imperfectives, en faisant varier le type aspectuel de la prédication. Nous montrons que, contrairement au français, le polonais permet, pour certains types de prédications, de distinguer formellement résultativité sémantique et résultativité pragmatique. Nous montrons également qu'a émergé, en polonais contemporain, une forme composée à mi-chemin entre une construction syntaxique et un paradigme flexionnel, qui n'est pas sans rappeler un état ancien du passé composé du français.

Dans nos analyses, nous utiliserons le terme de «situation» comme terme générique pour désigner le procès dénoté par la forme verbale, quelle que soit la nature de ce procès. 


\section{La résultativité et la valeur de parfait}

La résultativité est assurément une notion-clé en aspectologie. Différentes conceptions en ont été données, mais toutes associent généralement résultativité et valeur de parfait. Nous allons donc commencer par éclairer le rapport qu'il y a entre ces deux notions. Pour ce faire nous examinerons certains emplois du passé composé en français.

\subsection{Le passé composé et la valeur de parfait}

On partira du principe, certes discutable mais néanmoins assez généralement admis, que les emplois du passé composé français se subdivisent en deux types principaux, eux-mêmes susceptibles de distinctions plus fines (cf. par ex. Waugh 1987, Desclés et Guentchéva 2003) : un type aoriste, appelé parfois, depuis Benveniste (1966), « aoriste de discours » pour le distinguer du passé simple; et un type parfait, dit aussi «accompli» ou encore « présent résultatif». Seule la valeur de parfait nous intéresse ici.

Il convient de préciser qu'il s'agit de parfait du présent, puisque le terme de "parfait» désigne, au sens où nous l'entendons ici, non pas un temps mais une valeur aspectuelle indépendante du temps, et que le français possède trois parfaits : un parfait du présent (le passé composé à valeur de parfait), un parfait du passé (l'un des emplois du plus-que-parfait) et un parfait du futur (l'un des emplois du futur antérieur).

Les aspectologues s'accordent généralement pour reconnaître que ce qui fait la spécificité des formes verbales exprimant le parfait, c'est qu'elles renvoient simultanément à deux moments distincts. Ainsi pour Koschmieder (1929/1996), il y a valeur de parfait quand il y a

« un état suscité par l'accomplissement d'une action située dans le passé par rapport à cet état » $(1996: 27)$.

Reichenbach (1947), dans son modèle des trois «points », caractérise les tiroirs exprimant le parfait comme dissociant la référence temporelle (point of reference) et la situation dénotée par le lexème verbal (point of the event). Comrie (1976: 52) définit le parfait comme exprimant

« a relation between two time-points, on the one hand the time of the state resulting from a prior situation, and on the other the time of that prior situation $»$.

De son côté Guentchéva (1990 : 149), décrivant différentes valeurs du parfait en bulgare, distingue "l'état attribué au sujet de la relation prédicative » et "le processus qui lui a donné naissance », l'état étant adjacent et postérieur au processus. Karolak (1997) caractérise quant à lui le parfait comme une 
forme verbale dont le fonctionnement est fondamentalement inférentiel : le contenu visé par un parfait est présenté de façon indirecte, et le contenu explicitement formulé concerne une temporalité antérieure.

Comme on le voit, par delà les différentes caractérisations qui en ont été données, la valeur de parfait est toujours définie comme ayant pour propriété principale d'impliquer deux moments distincts.

\subsection{Principaux emplois de la valeur de parfait}

Ce fonctionnement bi-temporel se prête à toutes sortes d'emplois inférentiels. Mais ces emplois sont parfois difficiles à distinguer et la description de leurs effets pragmatiques assez subtile. Cependant nous voudrions essayer de montrer qu'il est possible de mettre un peu d'ordre dans cette diversité, en distinguant trois types inférentiels de parfait. Rappelons que nous ne nous intéresserons ci-dessous qu'à la valeur de parfait du présent, donc, s'agissant du français, au passé composé à valeur de parfait.

\subsubsection{Emplois illatif, abductif et explicatif}

Comme on va le voir, chacun de ces types correspond à un mode de contextualisation spécifique, implique un type d'inférence particulier et parfois une valeur évidentielle particulière.

(i) Nous distinguerons tout d'abord un emploi que nous appellerons illatif $^{1}$. Il s'agit du cas où la désignation de la situation vise à informer, non pas de la situation proprement dite, mais d'une conséquence de celle-ci. Autrement dit, de l'inférence situation $=>$ résultat, on ne retient pratiquement ici que le résultat. Ce dernier est valide et pertinent dans le contexte d'énonciation. Il s'agit du cas le plus prototypique de la valeur de parfait, celui qu'on désigne souvent par le terme d'«accompli» dans la tradition aspectologique française. Il est intéressant de noter que, selon les propriétés aspectuo-temporelles du prédicat verbal, cet emploi se manifeste tantôt de façon discrète tantôt de façon spectaculaire. De façon générale il se manifeste d'une façon particulièrement claire quand le prédicat verbal est de type transitionnel (au sens de Vet 1980) et/ou télique : le parfait est alors utilisé pour désigner, de façon quasi métonymique, l'état consécutif à la phase de transition, comme dans les exemples suivants.

Adjectif dérivé de illation, terme utilisé jadis par la tradition logicophilosophique pour désigner l'inférence. Nous l'entendons ici au sens prospectif, de la cause à l'effet. L'abduction en revanche est rétrospective. 
(1) - Mais je parle, je parle... quand vous avez des questions à me poser, peutêtre ?

- Heu... non.

- Mais vous êtes bien venu pour m 'interviewer? (S. Guitry, Quadrille, 42)

Je suis calme maintenant. Tout est fini, bien fini. Je suis sorti de l'horrible anxiété où m'avait jeté la visite du directeur. Car, je l'avoue, j'espérais encore. Maintenant, Dieu merci, je n'espère plus. (V. Hugo, Le dernier jour d'un condamné, 109)

Dans (1), vous êtes bien venu pour m'interviewer vaut pratiquement pour vous êtes ici pour m'interviewer, et dans (2), je suis sorti de l'horrible anxiété... vaut pour je ne suis plus dans l'horrible anxiété. Dans ces deux exemples, l'état désigné découle directement du sens même du verbe utilisé (venir, sortir) : respectivement 'être ici, être présent', et 'ne plus être dans l'horrible anxiété'.

(ii) Cependant la direction de l'inférence peut être inverse du cas précédent. Par exemple, après avoir constaté un certain état de choses, on peut inférer qu'une certaine situation, susceptible d'avoir produit cet état, a eu lieu. Nous parlerons alors d'emploi abductif. De façon caractéristique, l'énoncé a alors presque toujours une valeur conjecturale. Guentchéva (1990) appelle cet emploi «parfait de reconstruction $»^{2}$. En voici deux exemples :

(3) Maman savait tout faire et bien faire. Des herbes innombrables et des fleurs qui ornaient la surface de notre coin de terre, elle connaissait les vertus et les maléfices. Nous entendait-elle tousser: «Tu as de nouveau bu de l'eau à la fontaine alors que tu étais en transpiration... ». (M. Zermatten, Ô Vous que je n'ai pas assez aimée !, 110)

Pourtant il est ému et ses yeux sont rouges. Il a probablement pleuré mais je n’ose pas le lui demander. (J.-L. Pons)

Tu as de nouveau bu de l'eau à la fontaine doit s'entendre ici au sens de 'tu dois à nouveau avoir bu de l'eau à la fontaine' ou 'je soupçonne que tu as de nouveau bu de l'eau à la fontaine'. Cette valeur évidentielle particulière est inférée du contexte d'énonciation et des circonstances rapportées dans le texte : la mère, entendant ses enfants tousser, en infère qu'ils ont pris froid en buvant de l'eau à la fontaine. Mutatis mutandis la même analyse s'applique à

Le nom que nous avons donné à cet emploi s'inspire des analyses de cet auteur. Le terme d'abduction, introduit par Peirce, désigne un type d'inférence consistant, à partir d'un constat fait dans le contexte d'énonciation, à considérer l'objet de ce constat comme la conséquence d'une situation et d'en inférer à l'existence de cette situation. Il s'agit donc d'un raisonnement conjectural remontant de l'effet à la cause. Voir Peirce (1988), cité par Deledalle (1994 : $52)$. 
(4), où la modalité liée au raisonnement abductif est d'ailleurs explicitement formulée (cf. probablement).

Notons que ni l'inférence abductive ni la modalité de conjecture ne sont à proprement parler codées par le passé composé. Mais c'est bien le fonctionnement bi-temporel de la valeur de parfait qui rend possible ce type d'emploi et l'expression, dans ce contexte, de ce type d'inférence et de cette valeur évidentielle. Il existe cependant en français un tiroir qui a grammaticalisé cette valeur inférentielle-évidentielle, mais qui dans cet emploi est clairement marqué comme soutenu voire archaïsant : il s'agit du futur antérieur, tel qu'il est par exemple utilisé dans (5).

(5) Mais comment avez-vous chargé un Nubien de vous acheter une maison à Paris, et un muet de vous la faire meubler? Il aura fait toutes choses de travers, le pauvre malheureux. (A. Dumas, Le comte de Monte-Christo, 303)

(iii) Nous distinguerons enfin un emploi que nous appellerons explicatif. Il s'agit du cas où le locuteur a la connaissance du contenu des deux temporalités (par exemple parce qu'il a été témoin de la situation et qu'il en constate les conséquences actuelles), et où l'allocutaire n'a que la connaissance de l'état actuel. Le locuteur évoque alors la situation dans le but d'expliquer causalement l'état actuel pour l'allocutaire'. Dans l'exemple cidessous, le mouvement explicatif est monologique, mais cela ne change rien à la valeur explicative (au sens où nous l'entendons ici) du parfait.

(6) un morveux barbouillé se met à braire, sa tête a heurté le vaisselier, j'arrache les clés de leur crochet, je cours à la bagnole et on démarre en trombe, ce qui, en $2 \mathrm{CV}$, signifie pas grand-chose. (B. Blier, Les valseuses)

Le parfait sert ici à mettre en rapport une situation « antérieure » (sa tête a heurté le vaisselier), et une situation actuellement constatée (un morveux barbouillé se met à braire), construisant ainsi un rapport explicatif entre heurter le vaisselier et se mettre à braire. La proposition comportant le passé composé apporte ainsi rétrospectivement une explication à la situation décrite par la proposition précédente.

$\mathrm{Au}$ total, ces trois types inférentiels de parfait se différencient comme suit :

- Dans le cas d'un parfait en emploi illatif, l'expression vise à informer simultanément de la situation et de l'état actuel qui en découle. Assez souvent cependant, seul l'état résultant est visé, de telle sorte que la forme verbale a un fonctionnement quasi métonymique. Mais la saillance relative

L'emploi abductif comporte évidemment aussi une dimension explicative, mais l'explication est seulement conjecturale ; tandis que dans l'emploi explicatif stricto sensu, elle est assertée sur le mode de la certitude. 
de ces deux temporalités, plus exactement de leur contenu, peut varier de façon assez importante ${ }^{4}$.

- Dans le cas d'un parfait en emploi abductif, l'état résultant est lié à un constat fait dans la situation d'énonciation, et constitue donc une connaissance préalable; l'expression vise alors à informer, sur le mode conjectural, de la situation ou d'une situation pouvant être à l'origine de cet état et, en ce sens, pouvant l'expliquer.

- Dans le cas d'un parfait en emploi explicatif, le locuteur a la connaissance préalable et de la situation et d'un état actuel constaté. Son énonciation vise alors à asserter, mais cette fois-ci sur le mode de la certitude, l'existence d'un rapport causal, et en ce sens explicatif, entre ces deux informations.

Ces trois emplois se distinguent par leur valeur évidentielle. Il est probable que celle-ci est parfois marquée prosodiquement. Ils ont cependant en commun d'être des parfaits, c'est-à-dire d'impliquer chacun à leur manière deux temporalités.

\subsubsection{Le parfait d'expérience}

Toutefois un autre type de valeur interfère avec les distinctions exposées cidessus. Il s'agit de ce que Comrie (1976) a appelé le parfait « d'expérience » (nommé aussi parfait «existentiel» par McCawley 1971). On regroupe habituellement sous cette appellation divers emplois du parfait qui ont en commun le caractère temporellement indéfini de la situation désignée ${ }^{5}$. Par « indéfini », il faut entendre ici deux choses :

- D'une part, le fait que la forme verbale ne spécifie pas si la situation désignée s'est produite une ou plusieurs fois, cette spécification étant en quelque sorte laissée en suspens. L'essentiel est que la situation se soit produite une fois au moins.

- D'autre part, le fait que la situation désignée n'est pas localisée temporellement. S'agissant d'un parfait du présent, la seule chose qui est certaine est que la situation s'est produite dans le passé.

En voici deux exemples:

(7) Je refuse de nourrir mon python de souris vivantes, voilà, lui dis-je. C'est inhumain. Et il refuse de bouffer autre chose. Avez-vous déjà vu une pauvre petite souris face à un python qui va l'avaler? C'est atroce. (E. Ajar, GrosCâlin, 19, Frantext)

4 Cette caractéristique des parfaits avait déjà été signalée par Koschmieder: "l'accent porté sur l'action conduisant à l'état qu'elle a occasionné alterne souvent [...] avec l'accent porté sur l'état occasionné par l'action» (1996: 103). Sur ce point voir aussi Guentchéva (1990).

5 Leech (1971) appelle d'ailleurs le parfait d'expérience parfait « indéfini ». 
(8) - A 40 ans, avez-vous peur que les propositions de rôles déclinent?

- Non, pas du tout. Pour moi l'âge... [...] je sais pas ce que c'est l'âge... J'ai connu des hommes très vieux, des hommes moins vieux. J'ai connu des femmes plus belles à 60 ans qu'à 30. (interview de l'actrice S. Kimberlain, 2007, doc. internet)

On observera que dans ces exemples, les propositions comportant un passé composé peuvent être glosées au moyen de la formulation il est arrivé que... : (7) peut être glosé par 'vous est-il déjà arrivé de voir une pauvre petite souris face à un python qui va l'avaler?'; et (8) par 'il m'est arrivé de connaître des hommes très vieux... Il $m$ 'est arrivé de connaître des femmes plus belles à 60 ans qu'à 30'. Cette glose met en évidence d'une part la signification existentielle de l'énoncé concerné, d'autre part le caractère indéfini de la situation désignée. Par opposition, les autres types de parfaits peuvent être qualifiés de parfaits « définis ».

En français, certaines expressions ont un effet déclencheur plus ou moins décisif pour l'interprétation d'un parfait comme parfait d'expérience : déjà, un jour, toujours, souvent, une fois, jamais (au sens de 'une fois quelconque'), etc. Mais la présence d'une telle expression n'est pas indispensable, comme le montre $(8)^{6}$.

Notons que l'absence de localisation temporelle, donnée ci-dessus comme l'une des propriétés du parfait d'expérience, n'empêche pas une délimitation de la période à l'intérieur de laquelle la situation a eu lieu. Tel serait le cas dans ( $\left.7^{\prime}\right)$.

(7') Depuis que vous vous intéressez aux animaux, avez-vous déjà vu une pauvre petite souris face à un python qui va l'avaler?

Une autre caractéristique du parfait d'expérience est que le lien entre la situation évoquée et les conséquences visées est assez différent de celui qu'on observe avec les autres parfaits. Par exemple dans (7), il s'agit moins de demander à l'allocutaire s'il a effectivement assisté à l'événement décrit (la souris qui va être mangée par un python), que de lui demander s'il connaît les sentiments ou les émotions que ce spectacle peut susciter. La résultativité y est donc d'une tout autre nature que dans les exemples (1) à (6). L'appellation de parfait « d'expérience » rend plus ou moins bien compte du type de conséquence qui est ici visé : il s'agit de l'état actuel d'un sujet qui a fait telle ou telle chose, à qui il est arrivé un jour telle ou telle chose.

6 Dans certaines variétés du français, en particulier dans le domaine francoprovençal, le passé surcomposé a grammaticalisé cette valeur de parfait d'expérience. Pour une étude détaillée de l'emploi du surcomposé comme parfait d'expérience, voir Apothéloz (à par. $a$ et $b$ ). 
Cependant, on observe que la valeur de parfait d'expérience est transversale par rapport aux trois types inférentiels (illatif, abductif et explicatif) décrits plus haut. Ce qui veut dire qu'un parfait d'expérience peut être employé avec n'importe laquelle de ces trois valeurs. La chose est assez facile à montrer.

Imaginons qu'un ami me demande si je m'estime capable de faire un petit film documentaire sur le baptême de son fils. Pour justifier une réponse positive, je pourrais par exemple faire savoir à cet ami que j'ai une certaine expérience du cinéma et dire j'ai fait quelques documentaires. Je produirais alors un parfait d'expérience de type illatif. Imaginons maintenant que j'accepte de tourner ce film mais sans informer mon ami de mes expériences en matière de cinéma; et que, me voyant faire, et constatant ensuite le résultat, cet ami se montre surpris de mon savoir-faire. Il pourrait alors dire quelque chose comme : toi tu as fait du cinéma, ou simplement : tu as (déjà) tourné des films. Il produirait alors un parfait d'expérience de type abductif, avec la valeur évidentielle propre à cet emploi. Imaginons enfin qu'un autre ami assiste au tournage du documentaire en question et s'étonne de ma façon de faire, qu'il trouve très professionnelle. Le père de l'enfant baptisé, que j'avais informé de mon expérience en matière de cinéma documentaire, pourrait alors lui dire : il a fait des documentaires, produisant ainsi un parfait d'expérience de type explicatif.

Notre analyse de la typologie des valeurs de parfait est donc différente de celles de Comrie (1976) ou de Guentchéva (1990). Pour ces auteurs, en effet, le parfait d'expérience se situe, dans leur typologie, au même niveau que les autres types de parfaits - pour Guentchéva par exemple, au même niveau que le parfait abductif (qu'elle appelle « de reconstruction »). Ils le distinguent donc du parfait résultatif. Nous pensons au contraire que tous les parfaits sont, par définition, résultatifs, et que cette résultativité se décline selon deux dimensions: une première dimension, qui est celle de la définitude, et qui permet de distinguer des parfaits " définis » et des parfaits « indéfinis » (traditionnellement appelés «parfaits d'expérience »); et une seconde dimension qui est celle du type inférentiel, dimension à l'intérieur de laquelle nous avons distingué trois variétés : illatif, abductif et explicatif.

\subsection{Résultativité « sémantique » et résultativité « pragmatique »}

A examiner les exemples commentés dans la section précédente, on se rend compte que la notion de résultativité peut recouvrir des phénomènes extrêmement disparates. Cela est dû au fait que tous les verbes ne sont pas également prédisposés à produire de la résultativité. Pour ne prendre que quelques exemples, il est frappant de constater que des verbes comme arriver, fermer ou s'endormir se prêtent particulièrement bien à mettre en évidence la valeur de parfait lorsqu'ils sont fléchis à un temps composé. En 
discours ils sont régulièrement utilisés au passé composé pour signifier, respectivement, les états "être arrivé » (il est arrivé), "être fermé » (on l'a fermé) et "être endormi» (il s'est endormi). Il en va autrement de verbes comme courir, aimer ou heurter, dont les potentialités résultatives sont très différentes et, en un sens, moindres. Le paramètre qui est ici en cause est celui de la transitionnalité : les verbes arriver, fermer et s'endormir ont une signification typiquement transitionnelle et définissent par conséquent leur propre état résultant, ce qui n'est pas le cas de courir, aimer ou heurter, dont le sens ne comporte aucune idée de transitionnalité. Cela ne veut pas dire que ces verbes ne puissent pas, dans telle ou telle circonstance, être utilisés avec l'intention de signifier un état résultant. Mais quand cette situation se produit, l'état résultant n'a pas la même prévisibilité que lorsqu'il s'agit d'un verbe transitionnel. Par exemple on peut fort bien dire, en visant un état résultant, $i l$ a couru; mais l'état visé est alors toujours lié à des facteurs contingents. Dans cet exemple il pourrait correspondre à des informations variables et tributaires du contexte, comme: 'il est essouffle', 'il est en sueur', 'il est arrivé à l'heure', etc. L'énoncé il a couru peut être produit pour attirer l'attention sur des états de ce type, et par exemple pour en donner une explication.

Nous proposons donc de distinguer deux types de résultativité. Le premier type est celui auquel nous venons de faire allusion à propos des verbes arriver, fermer et s'endormir. Ces verbes comportent dans leur sens même un état résultant. Nous parlerons dans ce cas de résultativité sémantique. Le second type de résultativité est celui illustré par des verbes comme courir, aimer et heurter, dont la signification n'implique pas d'état résultant. Nous parlerons dans ce cas de résultativité pragmatique. Dans (1) et (2) ci-dessus (verbes venir et sortir), il s'agit typiquement de résultativité sémantique. Dans (4) et (6) (verbes pleurer et heurter), il s'agit de résultativité pragmatique.

Il faut toutefois se garder d'associer de façon trop rigide type de prédicat (transitionnel ou non transitionnel) et type de résultativité (sémantique ou pragmatique). S'il est vrai que les prédicats non transitionnels ne définissent pas sémantiquement un état résultant, et sont par conséquent inaptes à produire de la résultativité sémantique, les prédicats transitionnels peuvent fort bien, quant à eux, être utilisés avec une visée résultative pragmatique. C'est ce qui se passe dans (3): boire de l'eau froide est un prédicat transitionnel ; pourtant ce qui est visé, c'est bien ici un résultat inféré pragmatiquement (le fait de tousser). De même, on peut fort bien dire quelqu'un a ouvert la fenêtre non pas pour faire savoir que la fenêtre est actuellement ouverte, mais pour rendre compte par exemple du fait qu'il y a un courant d'air ; pourtant ouvrir est un verbe transitionnel. Le rapport entre la situation et l'état résultant est alors indirect, de l'ordre du probable seulement. 
De même il y a certaines affinités entre le type inférentiel du parfait et le type de résultativité qu'il produit (quand il ne s'agit pas du parfait d'expérience). Ainsi, les parfaits abductifs sont en principe associés à la résultativité pragmatique, ce qui est assez logique si on considère que le parfait abductif sert en général à exprimer une conjecture et non une certitude. Les exemples (3), (4) et (6) en sont une illustration. Les autres types inférentiels (illatif et explicatif) ne semblent pas avoir d'affinité particulière pour l'une ou l'autre résultativités.

Un cas un peu différent est celui du parfait d'expérience. Par définition ces parfaits mettent en œuvre un type particulier de résultativité (celui que vise à saisir le qualificatif «d'expérience»), qui est de nature clairement pragmatique. Pour cette raison ils ne sont pas sensibles au type aspectuel $d u$ prédicat verbal. N'importe quel type aspectuel de prédicat peut a priori donner lieu à ce type de parfait.

Nous allons maintenant examiner comment le polonais exprime la résultativité. Mais il convient tout d'abord de donner quelques informations générales sur le système aspectuel et temporel de cette langue.

\section{Le système verbal polonais}

Le polonais présente un système de temps verbaux qui, au premier abord, paraît extrêmement simple au regard du français ${ }^{7}$. En effet, outre le conditionnel et l'impératif, cette langue possède trois tiroirs flexionnels: présent, passé et futur. Mais cette apparente simplicité au plan des tiroirs est compensée par une morphologie aspectuelle d'une grande complexité au plan du lexique. Dans cette langue, en effet, la majorité des signifiés verbaux existent sous deux formes lexicales, traditionnellement dites perfective et imperfective. Seul un petit groupe de signifiés verbaux n'existent que sous une unique forme aspectuelle (perfectiva tantum, imperfectiva tantum). Mais tout verbe appartient nécessairement à la catégorie des perfectifs ou des imperfectifs.

Au plan morphologique, il existe divers affixes permettant de dériver une forme perfective d'une forme imperfective, et vice-versa. Pour les besoins de cet article il suffit d'indiquer que les affixes de perfectivation sont des préfixes, et ceux d'imperfectivation, des suffixes. Par exemple : burzyć ('détruire', forme imp.) et zburzyć ('détruire, forme perf.), dać ('donner', forme perf.) et dawać ('donner', forme imp.). Certains couples aspectuels sont supplétifs, par exemple: mówić ('dire', forme imp.) et powiedzieć ('dire', forme perf.).

Cependant ces faits lexicaux ne sont pas indépendants du système des tiroirs. Ainsi, les formes perfectives n'ont pas de présent ; plus exactement, la

Pour une présentation synthétique, voir par exemple Vater (1995). 
forme perfective d'un signifié verbal, fléchie au même tiroir morphologique que son double imperfectif, a une valeur de futur. Quant à la forme imperfective, elle a, pour le futur, une forme qui lui est propre: elle est composée, utilisant comme auxiliaire le futur du verbe być ('être').

La relation entre tiroir verbal et forme aspectuelle en polonais peut donc être résumée comme suit : le tiroir présent est nécessairement imperfectif, et l'opposition imperfectif $v$ s perfectif ne se manifeste qu'aux tiroirs passé et futur (cf. Tab. 1).

\begin{tabular}{|l|c|c|c|}
\hline & PASSÉ & PRÉSENT & FUTUR \\
\hline \hline IMPERFECTIF & burzyłem & burzę & będę burzyć \\
\hline PERFECTIF & zburzyłem & - & zburzę \\
\hline
\end{tabular}

Tab. 1. - Burzyć ('détruire' IMP.) et zburzyć ('détruire' PERF.).

Formes des $1^{\text {ère }}$ pers. sg. du passé, du présent et du futur.

En slavistique, les formes perfectives sont généralement décrites comme exprimant une situation de façon complète, menée jusqu'à son terme «naturel» (i.e. celui résultant de la signification du verbe); les formes imperfectives sont quant à elles décrites comme exprimant une situation incomplète, n'incluant pas le terme naturel de la situation désignée (cf. par ex. Kuryłowicz 1977). Ces caractérisations, ainsi que d'autres plus ou moins équivalentes, donnent régulièrement lieu à toutes sortes de polémiques, consistant par exemple à indiquer qu'il existe de nombreux contre-exemples à ces définitions, ou à signaler que tous les signifiés verbaux n'incluent pas nécessairement un terme naturel (ce qui ne les empêche pas d'avoir une forme perfective). Il est impossible, dans le cadre du présent article, d'entrer dans ce type de discussion. Nous nous contenterons de préciser ici que, de façon caractéristique, les formes imperfectives sont également utilisées pour signifier l'itérativité et l'habitualité, à la manière de l'imparfait en français.

Une des différences entre les systèmes des tiroirs polonais et français est donc qu'il n'y a pas, en polonais, de tiroir spécialisé dans l'expression de la résultativite $^{8}$. On pourrait penser, par exemple, que les formes perfectives au passé expriment une telle valeur. Mais nous verrons que ce n'est pas systématiquement le cas. Ces formes peuvent en effet être utilisées dans le récit, où elles ont une valeur d'aoriste et sont régulièrement (et fidèlement) traduites par des passés simples ou des passés composés aoristiques en français.

8 Voir toutefois section 4.6. ici même. 


\section{L'expression de la résultativité en polonais}

Cette section ne prétend pas faire le tour de la question, qui est des plus complexes. Nous nous limiterons ici à donner quelques repères en essayant de montrer l'intérêt descriptif que présentent les notions qui ont été posées plus haut. Pour ce faire nous examinerons tout d'abord le cas des prédications transitionnelles non duratives et duratives, puis celui des prédications non transitionnelles non duratives et duratives. Une prédication est transitionnelle si elle implique le franchissement d'une borne marquant le début ou la fin d'un état.

Le terme de «prédication » se justifie par le fait que dans nos analyses, nous préférons utiliser, comme unité de référence, l'ensemble de l'expression prédicative $^{9}$ et non le verbe seul, celui-ci étant souvent sous-déterminé relativement à la transitionnalité. Par exemple, une prédication construite avec le verbe manger est transitionnelle quand l'expression qui fonctionne comme second actant du verbe désigne un objet entier (manger une/la tartine, manger (toute) la viande), mais non transitionnelle quand cette expression désigne une partie d'objet, comme le fait par exemple un article partitif (manger de la viande). Cette distinction est essentielle en polonais, car elle détermine le choix de la forme verbale: perfective, quand l'expression du second actant désigne un objet entier, imperfective sinon (Wierzbicka 1967).

\subsection{Les prédications transitionnelles non duratives}

On observe ici la régularité suivante: quand la résultativité qu'il s'agit d'exprimer est purement sémantique, c'est la forme perfective qui est utilisée. La forme imperfective sert quant à elle à exprimer la résultativité pragmatique.

Voici tout d'abord quelques exemples comportant un verbe à la forme perfective (les formes concernées sont en gras) ${ }^{10}$.

(9) Przyjechaly wozy TV, pracują kamery. arriver.PERF.PASSÉ véhicules TV travailler.IMP.PRÉS. caméras Les véhicules de la télévision sont arrivés, les caméras tournent. (Kuszmider 1999 : 116)

9 Verkuyl \& Vet (2004) parleraient ici d'« aspect prédicationnel ».

10 Les gloses de la deuxième ligne utilisent les conventions suivantes: 'PASSÉ', 'PRÉS.' 'IMPÉR.' indiquent respectivement les temps passé, présent et impératif. 'IMP.' = imperfectif, 'PERF.' = perfectif, 'PREP.' = préposition, 'PP.' = participe passé, ' $\mathrm{N}$ ' = nom, 'PE.' = particule énonciative. Pour des raisons de lisibilité toutes les autres indications grammaticales (personne, genre, cas, etc.) sont délibérément omises. 

changer.PERF.PASSÉ travail...

J'ai changé de travail. Maintenant je travaille dans une grosse entreprise internationale.

$\begin{array}{llll}\text { Zobacz! } & \text { Ktoś } & \text { otworzyl } & \text { okno. } \\ \text { regarder. PERF.IMPÉR. } & \text { quelqu'un } & \text { ouvrir.PERF.PASSÉ } & \text { fenêtre } \\ \text { Regarde! Quelqu'un } & \text { a ouvert la fenêtre. } & \end{array}$

Dans ces exemples, l'énoncé vise à exprimer l'état résultant tel qu'il est déductible du signifié du verbe dans sa forme perfective. Les exemples (9) et (10) sont de type illatif. Dans (9) les véhicules de la télévision sont actuellement présents; dans (10) le locuteur ne travaille plus là où il travaillait. (11) pourrait, selon le contexte dans lequel il est produit, réaliser un parfait illatif, abductif ou explicatif. Quoi qu'il en soit il implique que la fenêtre est actuellement ouverte. Sur ce type d'exemple, voir aussi Włodarczyk (1994: 124).

Moyennant un contexte adéquat, les prédications de (10) et (11) se prêtent à une interprétation pragmatique de la résultativité si elles sont exprimées au moyen d'une forme imperfective du verbe.

$$
\begin{array}{ll}
\text { Witajcie. Była spora przerwa, ale rozumiecie, } \begin{array}{l}
\text { zmienialem } \\
\text { changer.IMP.PASSÉ }
\end{array} & \begin{array}{c}
\text { pracę. } \\
\text { travail }
\end{array} \\
\text { Ma być lepiej i za większą pensję. Się okaże. (doc. internet) } &
\end{array}
$$

Salut! Il y a eu une longue interruption, mais vous comprenez, j'ai changé de travail. Ça doit être mieux, un salaire plus élevé. On verra.

(13) [Le locuteur constate qu'il fait anormalement froid dans la pièce où il se trouve et, avant même d'avoir constaté si la fenêtre est fermée ou non, formule l'énoncé suivant:]

\begin{tabular}{lll} 
Ktoś & \multicolumn{1}{c}{ otwieral } & okno. \\
quelqu'un & ouvrir.IMP.PAssé & fenêtre \\
Quelqu'un & ouvert la fenêtre. &
\end{tabular}

Dans (12) le participant à un forum de discussion sur Internet excuse sa longue absence sur ce forum en écrivant qu'il a changé de travail. L'absence est traitée ici comme une conséquence pragmatique du changement de travail, et l'imperfectivité permet de lier explicativement la situation « changer de travail » à cet état de fait. Avec une forme perfective, l'énoncé n'aurait désigné que l'état consistant à avoir un nouveau travail et perdrait ainsi toutes ses vertus explicatives. Le choix de la forme imperfective permet également de désigner allusivement toutes les conséquences pratiques entraînées par un changement de travail. Dans (13), l'imperfectivité permet de mettre en rapport un constat indirectement lié à l'ouverture d'une certaine fenêtre, et cette ouverture. L'énoncé est produit dans un contexte clairement abductif. Contrairement à (11), il ne présume ni que la fenêtre est actuellement ouverte ni qu'elle est fermée (cf. pour un exemple analogue les 
commentaires de Kuszmider 1999: 115). On découvre que le polonais, à travers le choix grammatical de la perfectivité ou de l'imperfectivité, offre la possibilité de contextualiser la signification de l'énoncé.

Cependant il n'est pas possible de trouver un équivalent imperfectif pour (9). La raison est la suivante. La forme imperfective correspondant à przyjechać ('arriver', perf.), à savoir przyjeżdzać ('arriver', imp.), a lexicalisé une valeur itérative. Ce phénomène est assez fréquent en polonais, avec les verbes transitionnels non duratifs (voir notamment Laskowski 1998). Par exemple, les formes imperfectives suivantes sont également toujours itératives : znajdować ('trouver'), upadać ('tomber'), gubić ('perdre').

\subsection{Les prédications transitionnelles duratives}

Il s'agit des accomplissements au sens de Vendler (1957). La résultativité sémantique y est exprimée, comme pour les non duratifs, par la forme perfective du verbe.

$$
\begin{array}{lll}
\text { Zobacz, } & \text { co } & \text { narysowalem ! } \\
\text { regarder.PERF.IMPÉR. } & \text { ce que } & \text { dessiner.PERF.PASSÉ } \\
\text { Regarde ce que j'ai dessiné ! } &
\end{array}
$$

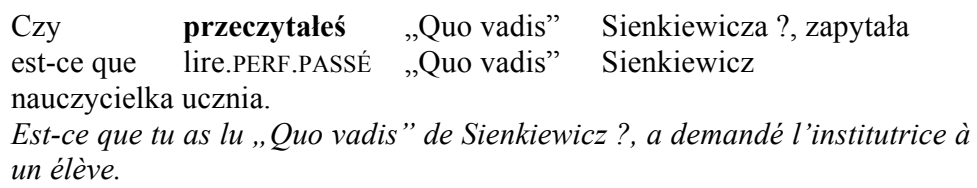

Dans (14) l'état résultant est matérialisé par l'existence de l'objet dessiné, dessiner étant un verbe «créatif». Dans (15) le verbe lire dans sa forme perfective désigne le parcours complet du livre en question. Le choix de cette forme indique par conséquent que la question porte bien sur la complétude de ce parcours.

Il convient de noter que la résultativité sémantique n'exclut pas la résultativité pragmatique. Ainsi, dans un contexte où le livre en question aurait été prêté à l'allocutaire, une question comme (15) pourrait très bien être posée pour lui demander s'il peut rendre ce livre, ou pour lui faire savoir qu'on voudrait qu'il le rende. Mais dans ce cas la résultativité " première » serait bien toujours sémantique.

La résultativité pragmatique est exprimée, comme pour les non duratifs, par la forme imperfective du verbe.

$$
\begin{array}{lll}
\text { Kto rysowal } & \text { tego } & \text { konia ? } \\
\text { qui dessiner.IMP.PASSÉ } & \text { ce } & \text { cheval } \\
\text { Qui a dessiné ce cheval? } &
\end{array}
$$


La question (16) pourrait par exemple être posée si le locuteur veut faire savoir qu'il a constaté que le dessin dont il est question présente une caractéristique particulière, par exemple d'être particulièrement bien ou mal fait $^{11}$. Pour ce qui est de (17), la forme imperfective du verbe lire (qui est celle de la traduction attestée), contrairement à la forme perfective, n'implique pas que le dossier a été lu jusqu'à la fin. L'énoncé vise seulement ici à faire savoir que le locuteur sait un certain nombre de choses à propos de la mère de l'allocutaire et que ces connaissances ont été acquises à partir de la lecture du dossier.

Comme on le voit, le point commun à tous les exemples d'imperfectivité examinés jusqu'ici est d'une part la non-expression (ou la non-explicitation) de la complétude de la situation, d'autre part le déclenchement d'inférences conduisant à signifier implicitement qu'il existe dans le contexte d'énonciation des conséquences indirectes de la situation exprimée. Un troisième point, également fréquent quoique non systématique, est que le contexte d'énonciation donne accès à des informations qui vont à l'encontre de ce que dit littéralement l'expression verbale: le dessin est terminé, le changement de travail a eu lieu, et pourtant ces situations sont décrites avec une forme signifiant en principe l'incomplétude ${ }^{12}$.

On notera que des questions comme qui a ouvert la fenêtre?, qui a fait ce dessin? etc., ont deux traductions en polonais. Soit la question porte uniquement sur l'identification de l'agent de la situation, et c'est la forme perfective du verbe qui est choisie; soit la question comporte, en plus, des allusions à des conséquences indirectes, à une caractéristique repérable dans le contexte d'énonciation (traces d'ouverture ou de tentatives d'ouverture de la fenêtre, dessin présentant une caractéristique particulière, etc.), et c'est alors la forme imperfective qui est utilisée.

$11 \quad$ Koschmieder (1929) utilise des exemples similaires pour défendre l'idée selon laquelle les notions de complétude et d'incomplétude ne permettent pas selon lui de rendre compte de la distinction perfectif $v s$ imperfectif. Sur ce point voir aussi Vater (1995).

12 On pourrait en déduire que les formes imperfectives signifient non pas l'incomplétude, mais seulement l'absence d'indication concernant la complétude. Telle est à peu près la thèse de Forsyth (1970). 


\subsection{Les prédications non transitionnelles non duratives}

Les prédications non transitionnelles se comportent de façon assez différente des prédications transitionnelles vis-à-vis de la résultativité et de l'opposition perfectif $v s$ imperfectif. Rappelons que, par définition, les prédications non transitionnelles ne peuvent pas produire de résultativité sémantique.

Les verbes non transitionnels non duratifs ont pratiquement tous une forme imperfective, mais celle-ci a presque toujours un sens itératif, comme certains verbes transitionnels non duratifs évoqués plus haut. Il en résulte que les prédications non transitionnelles non duratives ont toujours recours à une forme perfective pour désigner la résultativité pragmatique, en polonais.

$$
\begin{array}{lll}
\text { Przepraszam, jestem trochę spóźniona. } & \begin{array}{l}
\text { Spotkalam } \\
\text { rencontrer.PERF.PASSÉ copine }
\end{array}
\end{array}
$$

Je suis désolée, je suis un peu en retard. J'ai rencontré une copine.

$\begin{array}{ll}\text { Ty tutaj! } & \text { Spóźnilaś się na pociąg. } \\ \text { toi ici } & \text { rater.PERF.PASSÉ train } \\ \text { Toi ici! } & \text { Tu as raté le train. }\end{array}$

Dans (18) la forme verbale est de type explicatif, et dans (19) elle est de type abductif. Le type illatif est également possible. Imaginons par exemple que quelqu'un appelle l'ambulance pour faire part d'un accident: il pourrait produire un énoncé comme une voiture a heurté un piéton, en visant ainsi un élément actuel du contexte d'énonciation, par exemple qu'il y a un blessé.

\subsection{Les prédications non transitionnelles duratives}

Ces prédications désignent des activités ou des états. Elles se caractérisent par la durée et l'absence de transition. Leur interprétation résultative apparaît contextuellement quand elles sont utilisées dans des énoncés dont le sens est lié par inférence à un état de fait actuel. (20)-(21) illustrent les activités, et (22)-(23) les états.

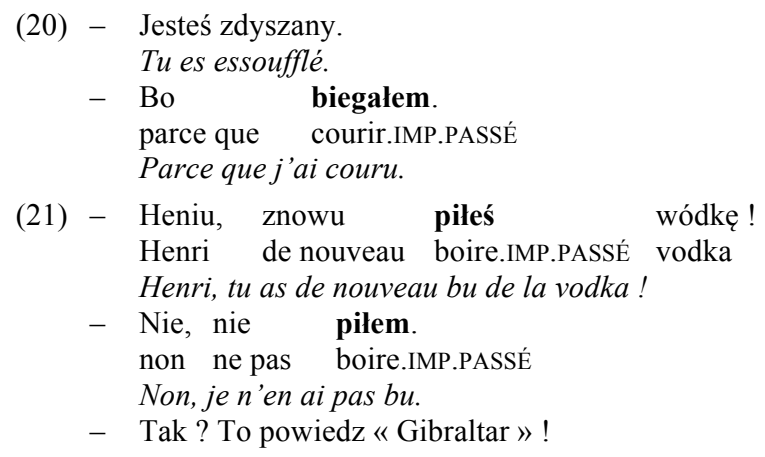


Ah bon? Alors dis « Guibraltar»!

- No dobra, pilem!

Bon d'accord, j'en ai bu! (doc. Internet)

(22) - Czemu wróciłeś tak szybko ?

Pourquoi es-tu rentré aussi vite?

- Bo, tęsknilem za tobą.

parce que languir.IMP.PASSÉ PRÉP. toi

- Parce que je languissais après toi.

(23) - Mizernie dziś wyglądasz.

Tu n'as pas l'air très bien aujourd'hui.

- No bo, chorowalem.

PE. parce que être malade.IMP.PASSÉ

- Parce que j'ai été / j'étais malade.

Toutes les formes verbales sont ici imperfectives. Elles sont de type explicatif dans (20), (22) et (23). Dans ces trois exemples un constat actuel est mis en rapport avec une situation passée: l'essoufflement, avec le fait d'avoir couru ; la rapidité du retour, avec l'ennui ; et l'apparence de la personne, avec le fait qu'elle a été malade. Le parfait est de type abductif dans la première occurrence de (21) : à partir de certains indices, on infère conjecturalement qu'une personne a bu de l'alcool. Les autres parfaits de (21) sont illatifs.

Pourquoi le polonais n'emploie-t-il pas ici des formes perfectives ? La raison en est que les formes perfectives des verbes utilisés dans ces exemples ne sont pas exactement, du point de vue sémantique, le pendant des formes imperfectives. De fait, les slavistes considèrent généralement que les verbes non transitionnels sont inaptes à former de vrais couples aspectuels, contrairement aux verbes transitionnels. On est donc ici en présence, une fois encore, de faux couples aspectuels.

La perfectivation des verbes non transitionnels duratifs peut avoir principalement, en plus de la perfectivité, trois sortes de conséquences sémantiques.

1. En premier lieu, elle peut produire un effet de délimitation temporelle. Il en va ainsi dans des couples comme biegać / pobiegać (respectivement 'courir' / 'courir pendant un court moment'), ou encore chorować / pochorować ('être malade' / 'être malade pendant une courte période', 'faire une courte maladie'). Comme le note Piernikarski (1969), on peut spécifier cette durée par des expressions comme un peu, une demiheure, etc.

2. En second lieu la perfectivation des verbes non transitionnels duratifs peut transformer le verbe en un verbe transitionnel. Deux cas doivent alors être envisagés, selon que le verbe transitionnel est inchoatif, ou égressif: chorować / zachorować ('être malade' / 'tomber malade'), kochać / zakochać się ('aimer' / 'tomber amoureux'), pour la valeur inchoative ; pić / wypić ('boire' / 'boire intégralement'), jeść / zjeść ('manger' / 'manger 
intégralement'), pour la valeur terminative. Parce qu'ils sont transitionnels, ces verbes peuvent produire de la résultativité sémantique.

3. En troisième lieu, la perfectivation des verbes non transitionnels duratifs peut produire l'idée qu'il y a extraction d'une "unité » de procès. C'est à peu près ce qui se passe en français avec des expressions comme « un coup de ", " un accès de », quand on oppose par exemple : avoir le cafard / avoir un coup de cafard, frapper / donner un coup, etc. Exemples polonais : dzwonić ('sonner') / zadzwonić ('donner un coup de sonnette'), tesknić ('languir') / zatęsknić ('avoir un accès d'ennui').

\subsection{Le parfait d'expérience}

La question du parfait d'expérience en polonais est des plus complexes. Nous ne pourrons, dans le présent article, que donner quelques indications sommaires.

En première approximation, on peut dire que le polonais emploie régulièrement la forme imperfective pour produire la signification de parfait d'expérience (Karolak 2007, et à par.). Les spécialistes du russe et du polonais abordent d'ailleurs souvent la question du parfait d'expérience dans le cadre d'un problème plus général qu'on peut formuler ainsi : comment se fait-il qu'on emploie parfois dans ces langues, pour désigner une situation dans le passé, une forme verbale imperfective alors que tout donne à penser que la situation est saisie dans son intégralité et jusqu'à son terme naturel (cf. Laskowski 1998, Bogusławski 2004, Stawnicka 2007) ? C'est donc d'abord sous la forme d'un paradoxe qu'apparaît la question du parfait d'expérience, paradoxe qui n'est pas sans rappeler celui de l'imparfait narratif du français (le parallélisme a d'ailleurs été formulé explicitement par Gebert 1992).

La question de (24) est un exemple de forme imperfective employée avec la valeur de parfait d'expérience.

$$
\begin{aligned}
& \text { Czy pileś kiedyś taką herbatę? } \\
& \text { est-ce que boire.IMP.PASSÉ une fois tel thé } \\
& \text { As-tu déjà bu un tel thé ? }
\end{aligned}
$$

Comme c'est le cas en français, le parfait d'expérience est souvent accompagné, en polonais, d'adverbiaux comme kiedyś ('une fois'), kiedykolwiek ('jamais', 'une fois quelconque'), już ('déjà'), nigdy ('jamais'), qui s'accordent avec le caractère indéfini de cette forme verbale et mettent en évidence l'interprétation expérientielle.

Cependant nous avons vu que beaucoup de verbes non duratifs polonais ont une forme imperfective qui a lexicalisé un sens itératif. Cette forme n'est donc pas disponible pour exprimer le parfait d'expérience. C'est alors la forme perfective qui est utilisée. Il s'agit par exemple des verbes znaleźć ('trouver', perf.), zgubić ('perdre', perf.) spotkać ('rencontrer', perf.), 
zakochać się ('tomber amoureux', perf.). Pour ces verbes le parfait d'expérience est marqué par des adverbiaux comme ceux cités ci-dessus ainsi que par divers indices contextuels.

\subsection{La construction mieć + SN + PARTICIPE PASSÉ}

Il existe cependant en polonais, en particulier en registre familier, une construction qui exprime spécifiquement l'état résultant. Elle se compose du verbe mieć ( 'avoir'), d'un syntagme nominal et d'un participe passé de forme perfective (le SN tantôt précède tantôt suit le participe). On ne la rencontre qu'avec les verbes transitifs. Elle semble suivre le schéma d'un attribut de l'objet, mais il ne s'agit en fait ni d'un vrai objet (du verbe avoir) ni d'un vrai attribut: le verbe mieć fonctionne plutôt ici comme une sorte d'auxiliaire. Cette construction est relativement répandue dans la langue parlée (Piernikarski 1969:148, Muryn, à par.). On en trouve de nombreuses attestations sur Internet. En voici deux exemples :

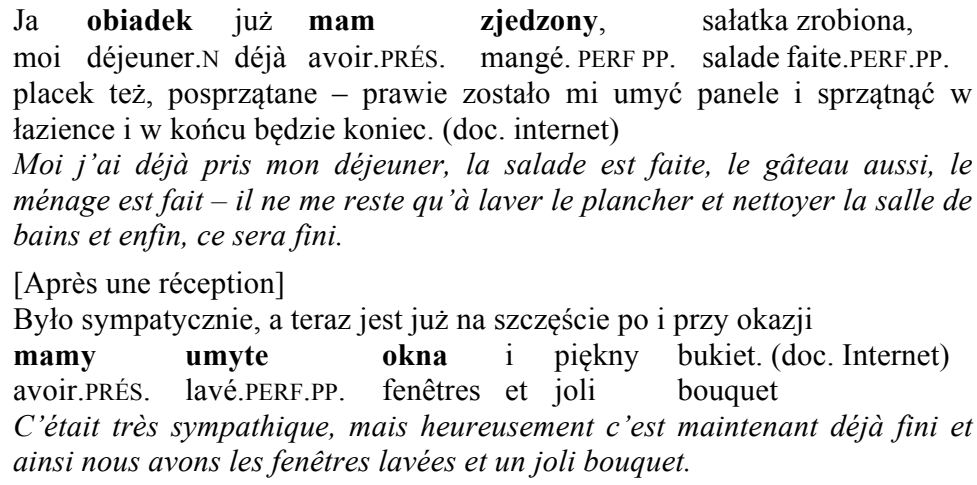

Dans (25), la construction qui nous intéresse est suivie d'une construction passive (sałatka zrobiona) avec omission du verbe être, sałatka étant au cas nominatif. L'exemple (26) est intéressant parce qu'il comporte une sorte d'anacoluthe : le verbe mieć ('avoir') y mis en facteur commun, d'une part avec la construction qui nous intéresse, d'autre part avec une construction transitive «normale » (avoir un joli bouquet). On observera que les deux prédications sont transitionnelles et que, en terme de type inférentiel, il s'agit de parfaits illatifs. Par ailleurs c'est clairement la résultativité sémantique qui est concernée ici.

Bien que son statut exact (flexion ou syntaxe) ne soit pas très clair, cette construction fait évidemment penser au passé composé français. Cependant elle s'apparente peut-être davantage, en français, à des constructions comme il a ses lunettes cassées, j'ai déjà cinq articles (d')écrits, etc., qu'à de vrais 
passés composés. Au plan de sa signification aspectuelle, elle est proche du present perfect anglais ${ }^{13}$. Signalons qu'elle se rencontre également au futur et au passé, produisant ainsi un futur et un passé résultatifs.

Indépendamment du problème de sa limitation à des verbes transitifs, la question se pose de savoir si cette forme périphrastique a les mêmes conditions d'emploi que les formes verbales synthétiques (i.e. les formes non composées du passé). Quand le sujet grammatical du verbe mieć ('avoir') coïncide avec l'agent du participe passé, la construction périphrastique coexiste avec la forme synthétique. Par exemple, la forme perfective du passé est possible dans (25). L'interprétation de l'énoncé permet chaque fois de déduire que le référent du morphème de personne du verbe mieć est aussi l'agent du verbe lexical représenté par le participe passé. En revanche, quand le lien entre ces deux éléments est moins clair, seule la forme périphrastique est employée. C'est le cas de (26). Dans cet exemple, en l'absence d'informations supplémentaires, rien ne permet de déterminer si l'agent du lavage des fenêtres coïncide ou non avec les individus désignés par la marque de personne -my ('nous') de verbe mieć. Dans chacun de ces exemples il est également possible d'employer la voix passive. En ce cas le verbe être peut être omis.

\section{Conclusion}

De multiples problèmes sont apparus au cours de cette étude, dont l'objet était certainement beaucoup trop vaste pour être traité dans le format d'un article. Au départ notre objectif n'était pas de comparer le français et le polonais. Pourtant les instruments conceptuels que nous avons mis en place dans la première partie (typologie des valeurs de parfait fondée sur des propriétés inférentielles, distinction entre résultativité sémantique et pragmatique) nous paraissent rétrospectivement fournir une excellente base pour entreprendre une étude contrastive de la grammaire de l'aspectuotemporalité de ces deux langues. A cet égard, deux points nous semblent particulièrement importants.

En premier lieu, il est frappant de constater à quel point les notions de perfectivité et d'imperfectivité recouvrent des phénomènes et des fonctionnements différents dans les deux langues. Une fois encore en aspectologie, on constate que la terminologie est pleine de chausse-trapes.

En second lieu, nous avons observé qu'avec les prédications transitionnelles, l'opposition perfectif $v s$ imperfectif était systématiquement

13 Selon Kuryłowicz (1987), le present perfect à l'époque de son apparition admettait lui aussi une relative liberté de l'ordre des éléments, notamment de l'objet relativement au participe. Il en va de même en ancien français, du moins avant le XIII ${ }^{\mathrm{e}}$ s. (cf. Marchello-Nizia 1999). 
utilisée en polonais pour contraster une résultativité sémantique et une résultativité pragmatique, chose que ne peut pas faire le français. Pour ce type de prédication, cette opposition permet notamment, en polonais, d'affiner le lien qui doit être établi entre le contexte immédiat de la parole et la situation désignée par le prédicat verbal, et donc de guider l'interprétation in situ de l'énoncé.

\section{Références}

Apothéloz, D. (à paraître, a). Le passé surcomposé et la valeur de parfait existentiel, Journal of French Language Studies.

Apothéloz, D. (à paraître, b). Le passé surcomposé existentiel, Etudes romanes de Brno.

Benveniste, E. (1966). Les relations de temps dans le verbe français, in : Problèmes de linguistique générale 1, Paris : Gallimard, 237-250.

Bogusławski, A. (2004). Aspekt i negacja, Warszawa : Wydawnictwo TAKT.

Comrie, B. (1976). Aspect. An introduction to the study of verbal aspect and related problems, Cambridge : Cambridge University Press.

Deledalle, G. (1994). Charles S. Peirce. Les ruptures épistémologiques et les nouveaux paradigmes, Travaux du Centre de recherches sémiologiques $62: 51-66$.

Desclés, J.-P.; Guentchéva, Z. (2003). Comment déterminer les significations du passé composé par une exploration contextuelle?, Langue française $138: 48-60$.

Forsyth, J. (1970). A grammar of aspect. Usage and meaning in the Russian verb, Cambridge : Cambridge University Press.

Gebert, L. (1992). Osservazioni sull'imperfettivo per esprimere fatti compiuti in lingue slave e romanze, in : W. Banyś ; L. Bednarczuk ; K. Bogacki (éds), Etudes de linguistique romane et slave, Kraków: Universitas, 217-226.

Guentchéva, Z. (1990). Temps et aspect: l'exemple du bulgare contemporain, Paris : Editions du CNRS.

Karolak, S. (1997). Le temps et le modèle de H. Reichenbach, Etudes cognitives / Studia kognitywne 2 : 95-125 (Varsovie : SOW).

Karolak, S. (2007). Składnia francuska o podstawach semantycznych, Kraków : Collegium Columbinum.

Karolak, S. (à paraître). Remarques sur l'équivalence du passé imperfectif polonais et des temps passés en français, Verbum.

Koschmieder, E. (1929). Zeitbezug und Sprache. Ein Beitrag zur Aspekt- und Tempusfrage. Leipzig/Berlin. Trad. franç.: Les rapports temporels fondamentaux et leur expression linguistique. Contribution à la question de l'aspect et du temps, Villeneuve-d'Ascq: Presses Universitaires du Septentrion, 1996. 
Kuryłowicz, J. (1977). Problèmes de linguistique indo-européenne, Wrocław, Warszawa, Kraków, Gdańsk : Ossolineum.

Kuryłowicz, J. (1987). Studia językoznawcze, Warszawa : Państwowe Wydawnictwo Naukowe.

Kuszmider, B. (1999). Aspect, temporalité et modalité en polonais et en français, Gap, Paris : Ophrys.

Laskowski, R. (1998). Kategorie morfologiczne - charakterystyka funkcjonalna, in : R. Grzegorczykowa ; R. Laskowski ; H. Wróbel (éds), Gramatyka współczesnego języka polskiego. Morfologia, Warszawa : Wydawnictwo Naukowe PWN, 147-172.

Leech, G.N. (1971). Meaning and the English Verb, London : Longman.

Marchello-Nizia, C. (1999). L'accord du participe passé avec l'objet direct en ancien français, Verbum $21, \mathrm{n}^{\circ} 3: 323-338$.

McCawley, J.D. (1971). Tense and time reference in English, in: C.J. Fillmore; D.T. Langendoen (eds), Studies in linguistic semantics, New York : Holt, Rinehart and Winston, 96-113.

Muryn, T. (à paraître). Le passé composé en fonction de parfait nontestimonial. Une analyse contrastive français-polonais. Actes $d u$ colloque "L'Europe des langues et la globalisation communicationnelle: enrichissement et menaces pour les langues ", Université de Gdańsk, 21-22 juin 2007.

Peirce, C.S. (1986). Writings of Charles S. Peirce, vol. 3 (M. Fisch, C. Kloesel et al., eds), Bloomington : Indiana University Press.

Piernikarski, C. (1969). Typy opozycji aspektowych języka polskiego na tle stowiańskim, Wrocław : Ossolineum.

Reichenbach, H. (1947). Elements of symbolic logic, London \& New York: Macmillan.

Stawnicka, J. (2007). Aspekt - iteratywność - określniki kwantyfikujace (na materiale form czasu przeszłego w języku polskim), Katowice : Wydawnictwo Uniwersytetu Śląskiego.

Vater, H. (1995). The tense system of Polish, in : R. Thieroff (ed.), Tense Systems in European Languages, vol. II, Tübingen: Max Niemeyer, $153-165$.

Vendler, Z. (1957). Verbs and time. Philosophical Review 66: 143-160. Repris dans: Z. Vendler, Linguistics in Philosophy, Ithaka, NY : Cornell University Press, 1967, 97-121.

Verkuyl, H. ; Vet, C. (2004). Tense and aspect in sentences, in : H. de Swart ; F. Corblin (eds), Handbook of French Semantics, Stanford: CSLI Publications, 235-279.

Vet, C. (1980). Temps, aspects et adverbes de temps en français contemporain, Genève : Droz.

Waugh, L.R. (1987). Marking time with the passé composé : toward a theory of the perfect, Linguisticae Investigationes 11, $\mathrm{n}^{\mathrm{o}} 1: 1-47$. 
Wierzbicka, A. (1967). On the Semantics of the Verbal Aspect in Polish, in : To Honor Roman Jakobson. Essays on the Occasion of His Seventieth Birthday, The Hague-Paris : Mouton, 2231-2249.

Włodarczyk, H. (1994). L'aspect verbal slave et les domaines du donné et du nouveau, Etudes cognitives / Studia kognitywne 1 : 113-130 (Varsovie : SOW). 